\title{
Transport de la neige par le vent sur un site de montagne : mesures et modélisation numérique à l'échelle du couloir
}

\author{
Drifting snow on a mountain site : measurements \\ and numerical modelling at avalanche path scale \\ par Jean-Luc Michaux, Florence Naaim-Bouvet, Mohamed Naaim \\ Division ETNA Cemagref
}

\begin{abstract}
Drifting snow is a serious problem, since it produces snowdrift on roads, and it is responsible of most avalanches in mountains, by creating wind slabs. In order to fight against this phenomena, the Etna unit of Cemagref, and the Snow Studies Center of Météo France have been working on snow drift for ten years, on the experimental site of Lac Blanc Pass. This site was developed thanks to the financial support of the Rhone-Alps Region, and with the logistic support of SATA (Alpe d'Huez ski resort company). It is a kind of natural cold wind tumnel, with dominant winds from the north to the south. During the last two years, we have installed on this site many sensors such as anemometers, snowdrift acoustic sensors, and snow poles to measure the height of snow. The aim was to create a data basis used to perform our knowledge concerning drifting snow, and to test the numerical model of drifting snow developed by the team [1].

This paper describes the results of the last two years. In particular, we present the analysis of the data of the drifting snow acoustic sensor, which allow us to determine threshold velocities, and to establish preliminary relations between drifting snow flows and the signal on the sensor. Then, thanks to an analysis of gust factors, both for the wind and for the snowdrift, we will show that most important drifting snow episodes occur during a period of relatively high regular wind, and not during period of high wind gusts. Lastly, we present a comparison of the results coming from our numerical model, and the in situ snow depth, for a drifting snow event.
\end{abstract}

\section{INTRODUCTION}

Grâce au support financier de la région Rhône-Alpes et au soutien logistique de la SATA. la division ETNA du Cemagref de Grenoble et le Centre d'Etudes de la Neige étudient le transport de neige par le vent sur le site expérimental du Col du Lac Blane depuis une dizaine d'années. Ce site, relativement plat et situé à $2700 \mathrm{~m}$ sur le domaine skiable de l'Alpe d'Huez, se comporte comme une soufflerie naturelle avec des vents dominants de secteur sud et de secteur nord. L'un des objectifs des expérimentations menées ces deux dernières années était de tester le modèle numérique de transport de neige par le vent développé par l'équipe [1]. Ce modèle est destiné à simuler les zones de dépôt et d'érosion de la neige à l'échelle d'un couloir d'avalanche. Nous présenterons successivement dans cet article, le site expérimental ainsi que les différents résultats obtenus, en insistant tout particulièrement sur les mesures issues du capteur acoustique de transport tout en introduisant la notion de coefficient de rafales pour le vent et le transport de neige par le vent. Enfin, nous comparerons, pour un épisode de transport donné, les résultats du modèle numérique et les mesures de hauteur de neige observées in situ.

\section{D DESCRIPTION DU SITE EXPÉRIMENTAL}

Afin d'étudier la distribution tri-dimensionnelle de la hauteur de neige durant l'hiver et de déterminer ainsi les zones de dépôt et d'érosion de la neige, nous avons installé deux réseaux de 25 perches à neige. Les résultats présentés dans cet article ne concernent que le site dit de "La Muzelle", qui est localisé au sud du Col : dans ce cas précis, 20 perches disposées suivant un maillage régulier dans la zone de dépôt sont complétées par un profil longitudinal $(200 \mathrm{~m})$ de 5 perches suivant l'axe Nord-Sud dans la zone d'érosion. Les hauteurs de perches sont relevées manuellement toutes les semaines, voire plus en cas d'épisodes de transport. A cette occasion, un sondage de battage et une détermination du profil stratigraphique du manteau neigeux sont effectués. Sur le site, des enregistrements continus de paramètres météorologiques classiques (intensité et direction du vent, température, précipitation) complètent ces mesures. Cependant toutes ces données météorologiques ne sont pas suffisantes pour tester un modèle numérique de transport de neige par le vent : le débit des particules de neige transportées ainsi que 
la vitesse seuil d'arrachement des particules sont des données d'entrée nécessaires au modèle. C'est pourquoi la division ETNA a développé en collaboration avec la société grenobloise Hydroémac un capteur de transport de neige par le vent basé sur un principe acoustique. Il s'agit d'un microphone miniature large bande placé dans un tube creux en aluminium d'une hauteur variable qui est lui-même exposé au flux de particules. Les sons dus aux impacts des particules sont convertis en signaux électriques par un sonomètre sélectif en fréquence : un filtrage passe-bande élimine les basses fréquences dues au vent et les hautes fréquences hors de la gamme audible. La valeur efficace de ce signal est convertie en tension continue. Six de ces capteurs ont été installés sur le site de La Muzelle.

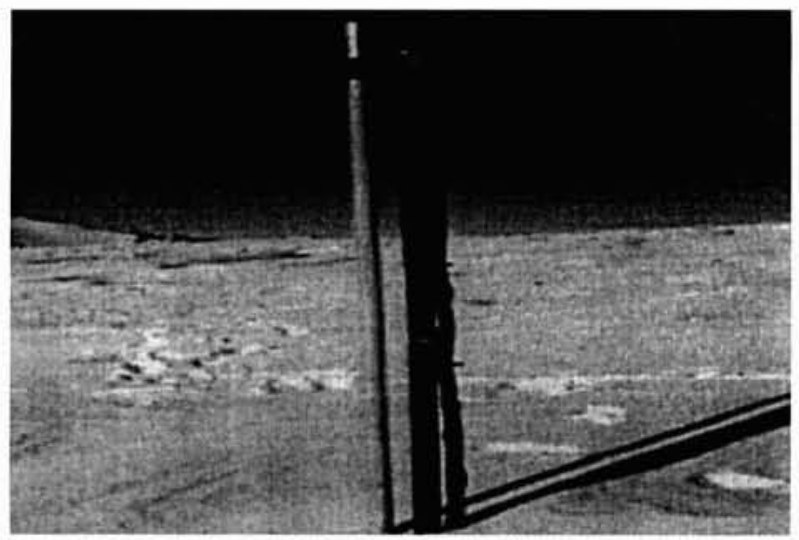

1. Le capteur acoustique de transport.

\section{III — LES DONNÉES RELATIVES À L'HIVER 98-99}

\subsection{Le site de la Muzelle : un site soumis} au transport de la neige par le vent

Comme mentionné précédemment, le site de la Muzelle est essentiellement soumis à des vents de secteur nord et de secteur sud (cf. figure 2). L'enneigement et les vitesses de vent importantes sont favorables au transport de la neige par le vent. En considérant qu'un signal de sortie de $50 \mathrm{mV}$ du capteur acoustique caractérise le début d'un épisode de transport de neige, $40 \%$ du mois de février est sujet au transport de neige.

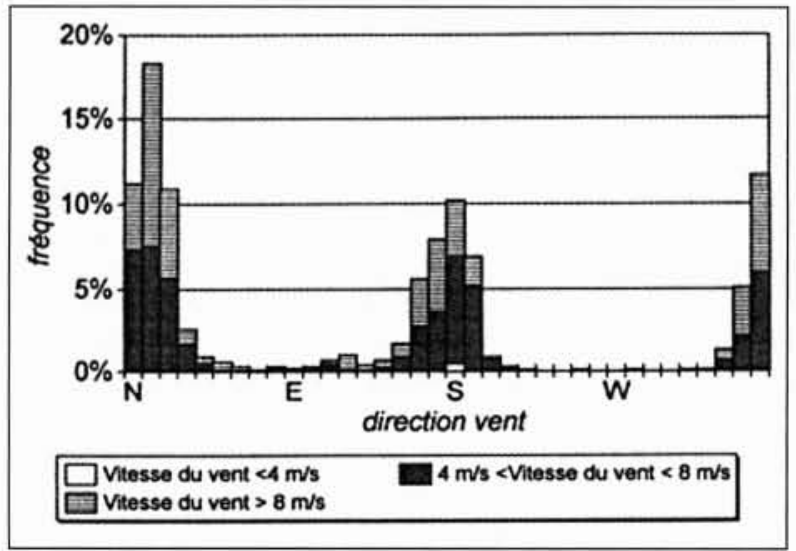

2. Rose des vents sur le site de la Muzelle durant I'hiver 1998-1999.

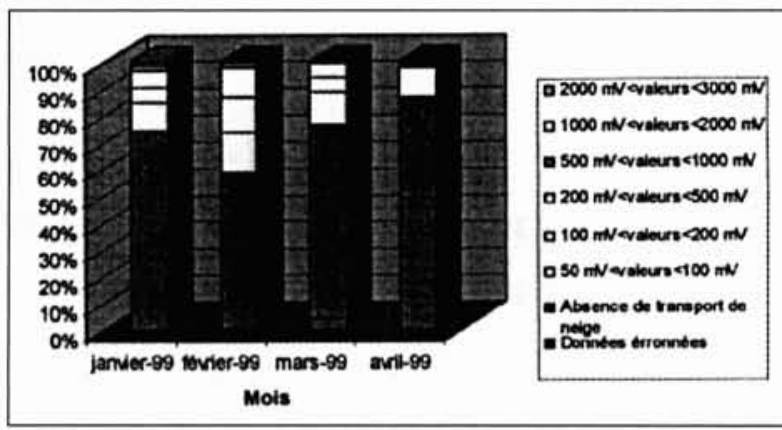

3. Occurrence des épisodes de transport.

NB: La tension issue du capteur acoustique de transport est représentative du débit de particules (voir paragraphe suivant).

\subsection{Le capteur acoustique de transport : des données encore incomplètes}

\subsubsection{Relation entre le débit de neige transportée et le signal enregistré par le capteur acoustique}

Les impacts des particules de neige sur le capteur acoustique produisent un son, son qui est transformé en une tension enregistrée sur une centrale d'acquisitions. Pour pouvoir utiliser les données enregistrées comme entrée du modèle numérique, il est nécessaire de relier la tension à un débit de particules. Nous avons donc comparé le débit de particules moyenné sur 15 minutes avec la tension enregistrée sur le capteur durant la même période et ce pendant plusieurs épisodes de transport. Le débit de particules est déterminé à partir de capteurs mécaniques de transport. Ces capteurs mécaniques, aussi surnommés "filets à papillons", sont constitués d'un support mécanique de $15 \mathrm{~cm} * 2 \mathrm{~cm}$ sur lequel est attaché un sac en nylon. Le piège qui est placé face à la direction du vent dominant est fixé à différentes hauteurs à côté du capteur acoustique. Le mélange air + particules pénètre dans le filet qui ne retiendra que la neige. Le poids de la neige est ensuite mesuré. Le bruit enregistré dépend de la hauteur du manteau neigeux sur le capteur acoustique ; c'est pourquoi ce dernier est fixé sur un mât de telle sorte qu'il puisse être rehaussé pendant la période hiver-

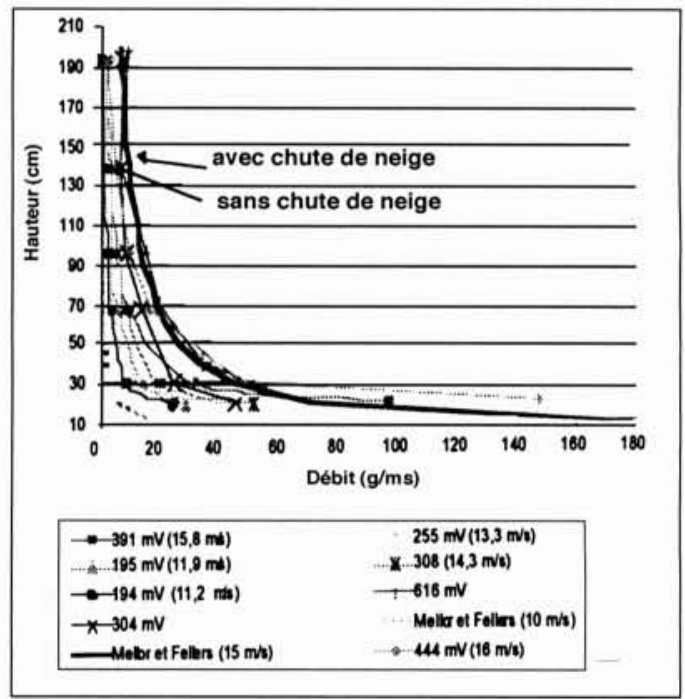

4. Relation entre le débit de particules et le signal enregistré par le capteur acoustique. 
nale (voir photographie 1). Durant le calibrage du capteur, ce dernier était situé dans une zone d'érosion, complètement dégagée de neige. Les résultats sont présentés sur la figure 4 . Ces mesures correspondent à deux épisodes de transport de neige (avec et sans chute de neige simultanée). On constate tout d'abord, que ces données sont en bon accord avec les formulations empiriques de Mellors et Fellers [2], bien que ces derniers ne distinguent pas les effets des chutes de neige lors du traitement de leur donnés [3]. Ici, cependant, les effets de la chute de neige simultanée sont visibles : en cas de chute de neige, le débit de particules au dessus de 1,3 mètre est quasi-constant et équivalent à $8 \mathrm{~g} / \mathrm{m}^{2} \mathrm{~s}$. Le faible nombre d'expériences réalisées ainsi que la faible plage de vitesse de vents enregistrés ne permet pas d'effectuer un calibrage complet. Il est nécessaire de poursuivre cet étalonnage pendant plusieurs campagnes de mesures.

\subsubsection{Influence du type de particules sur le capteur acoustique de transport}

Les différentes courbes ci-après représentent le bruit du capteur enregistré (moyenne sur $15 \mathrm{~min}$ en fonction de la vitesse du vent) pour différents épisodes de transport de neige et donc différents types de neige. Le modèle SAFRAN [4]-CROCUS [5] a été utilisé pour simuler les évolutions du manteau neigeux sur le site expérimental et déterminer ainsi le type de neige de surface.

Lors de l'épisode du 4 mars, on observe une chute de neige simultanée qui se traduit sur la courbe par une grande dispersion des points (cf. figure 5). En effet, l'intensité de la chute de neige est décorrélée de la vitesse du vent. Cette dispersion des points de mesures durant les épisodes de transport de neige avec chute de neige simultanée est une constante au cours de l'hiver (cf. figure 5).

L'épisode du 8 mars (cf. figure 5) correspond à une reprise de neige fraîche tandis que celui du 17 au 20 mars (cf. figure 5) correspond à une reprise de grains fins et celui du 16 au 18 janvier à une reprise de faces planes. Il semble que le bruit généré par les grains fins soit plus important que celui généré par les faces planes. Cependant la hauteur de la couche de neige au droit du capteur a varié au cours du mois de mars suite à des chutes de neige sans vent. Aussi, la différence de comportement entre les deux types de grains est-elle moins flagrante pour un autre capteur où la variation de la hauteur de neige a été moindre. Lors d'une utilisation opérationnelle, il sera indispensable d'adjoindre un capteur de hauteur de neige au capteur acoustique pour pouvoir corriger les données par rapport à la situation idéale où le capteur a été calibré (absence de manteau neigeux sur une zone d'érosion).

Les épisodes du 16 au 18 janvier et du 3 mars correspondent à des épisodes de reprise de particules de type faces planes. On remarque que ces deux courbes sont relativement semblables, la tension enregistrée étant grossièrement proportionnelle au cube de la vitesse. Cela ne va pas sans rappeler les formulations empiriques montrant que le débit de transport est proportionnel au cube la vitesse (Dyunin A. K. et Kotlyakov (1980)). Cela laisserait à penser que le débit de transport est proportionnel à la tension enregistrée sur le capteur acoustique. Le faible nombre de mesures réalisées pour l'étalonnage ne permet pas encore de le confirmer. Néanmoins les essais préliminaires du capteur de transport avaient montré que le signal généré par une quantité $\mathrm{Q}$ de sable lâché d'une hauteur $\mathrm{H}$ était le double de celui généré par une quantité de sable $(\mathrm{Q} / 2)$.

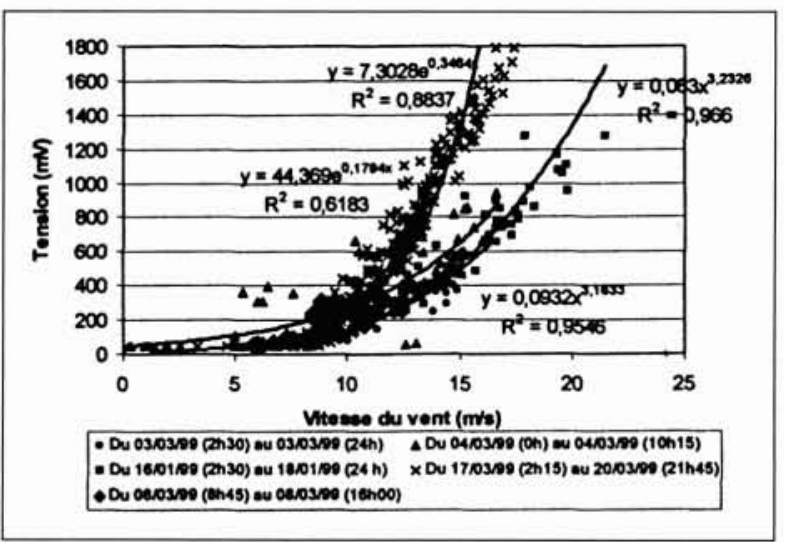

5. Tension moyenne sur $15 \mathrm{~min}$ (données issues du capteur acoustique $\mathrm{n}^{\circ} 5$ ) en fonction de la vitesse moyenne sur $15 \mathrm{~min}$ pour différents épisodes de transport de neige.

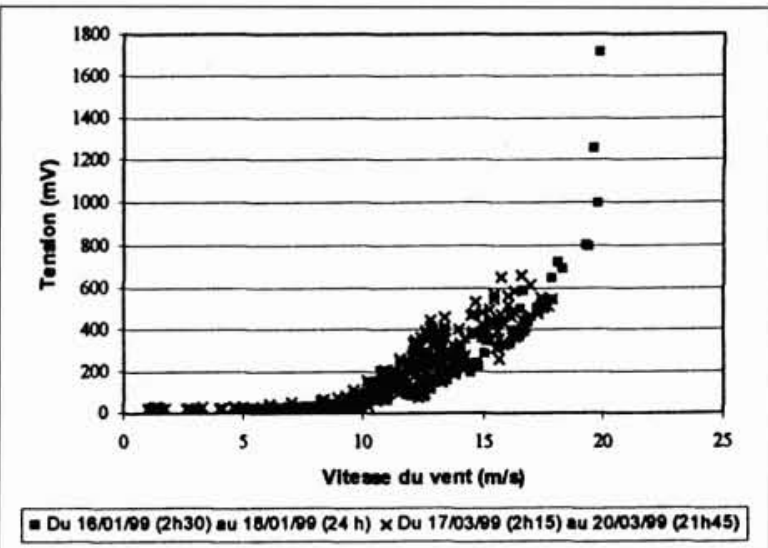

6. Tension moyenne sur 15 min (données issues du capteur acoustique $n^{\circ} 1$ ) en fonction de la vitesse moyenne sur 15 min pour différents épisodes de transport de neige.

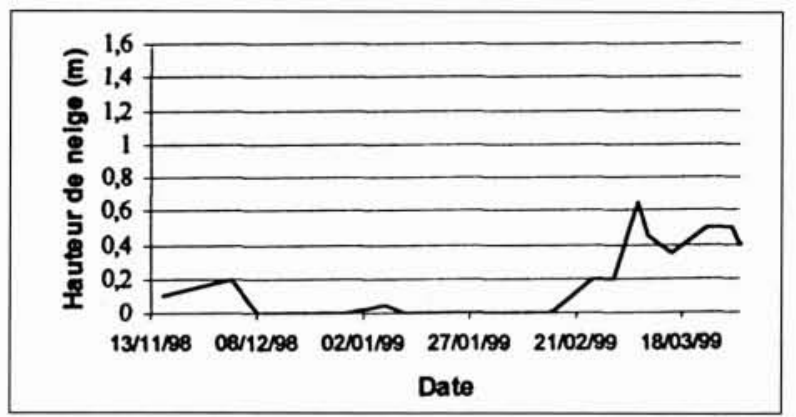

7. Evolution de la hauteur de neige au niveau du capteur de transport $n^{\circ} 5$.

\subsubsection{Détermination de la vitesse seuil}

Pendant la saison hivernale 1998-1999, cinq épisodes de transport de neige ont été sélectionnés. La vitesse moyenne d'arrachement des particules de neige a été déterminée grâce aux données issues du capteur acoustique et de l'anémomètre. Comme explicité précédemment, la chaîne SAFRANCROCUS a été utilisée pour simuler l'évolution du manteau neigeux sur le site expérimental. Grâce à cette modélisation, nous avons pu associer la vitesse seuil d'arrachement des particules à un type de grains. Dans le modèle SAFRANCROCUS, les particules de neige sont décrites par l'intermé- 
diaire de la dendricité (d comprise entre 0 et 1), le sphéricité ( $\mathrm{s}$ comprise entre 0 et 1 et la taille du grain gs (en mm).

Guyomarch et Merindol [7] estiment la vitesse seuil d'arrachement des particules à partir de la formulation suivante :

$$
U t_{(10 m)}=\frac{-\ln \left(\frac{d_{i}+1}{2,868}\right)}{0,085}
$$

avec $d_{i}$ indice de mobilité et $U_{t(10 \mathrm{~m}) \text {, vitesse moyenne }}$ horaire à 10 mètres de haut).

L'indice de mobilité est déterminé de la façon suivante :

Si $d>0 \quad d_{i}=0,75 d-0,5 s+0,5$

Si $d<0 d_{i}=0,583 \mathrm{gs}-0,833 s+0,833$

Sur la figure 8 , la ligne continue représente l'équation 1 et les points représentent nos données expérimentales. Jusqu'à présent. le modèle SAFRAN CROCUS ne prend pas en compte les épisodes de transport de neige, c'est-à-dire que les couches de neige de surface susceptible d'être érodée restent en place. Une évolution du modèle (SYTRON), permettant de prendre en compte ce phénomène, est en cours de développement. Malgré cela, on peut constater que nos données expérimentales sont en bon accord avec l'équation 1. Dans la plupart des cas, la vitesse d'arrachement seuil déterminée à l'aide du capteur acoustique est inférieure à celle déterminée par l'équation 1 , ce qui peut s'expliquer par l'importante sensibilité du capteur (un épisode de transport de neige est immédiatement détecté).

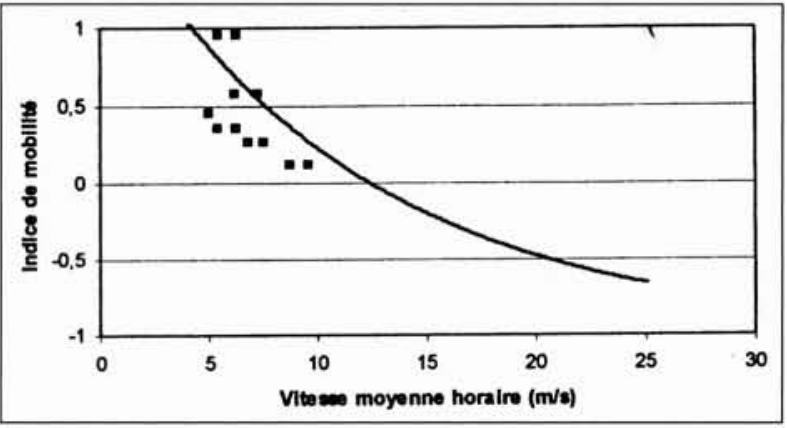

8. Vitesse moyenne horaire d'arrachement des particules en fonction de l'indice de mobilité des particules.

Lors d'un épisode de transport avec chute de neige simultanée, la vitesse moyenne horaire d'arrachement détectée par le capteur acoustique varie de $1,3 \mathrm{~m} / \mathrm{s}$ à $2,6 \mathrm{~m} / \mathrm{s}$ en fonction de l'intensité de la chute de neige.

\subsection{Le transport de la neige par le vent : un phénomène non stationnaire}

Jusqu'à présent, la modélisation du vent et du transport de neige par le vent ne prenait en compte que des valeurs moyennes. Par conséquent il nous a semblé intéressant de mettre en évidence l'aspect non stationnaire de ces phénomènes. Pour ce faire, nous avons étudié le coefficient de rafale, à la fois pour le vent et pour le transport de neige par le vent. Ce coefficient se définit comme le rapport de la valeur maximale sur la valeur moyenne du signal, pour un intervalle de temps donné.

\subsubsection{Coefficient de rafales du vent}

Les données obtenues sur le site expérimental de La Muzelle ont été traitées en trois étapes.
Tout d'abord, nous n'avons conservé que les données correspondant à un vent moyen supérieur à $4 \mathrm{~m} / \mathrm{s}$. En effet, pour une vitesse de vent plus faible que $4 \mathrm{~m} / \mathrm{s}$, nous avons considéré qu'il n'y avait pas de transport de neige par le vent ou très peu (cf. figure 8 ). De plus, une faible vitesse de vent génère souvent un gros coefficient de rafale qui n'a pas d'intérêt physique par rapport au problème du transport de neige par le vent. Par exemple, un coefficient de rafale de 5 obtenu par un vent moyen de $0,5 \mathrm{~m} / \mathrm{s}$ et une rafale de vent de $2.5 \mathrm{~m} / \mathrm{s}$ diffère totalement du même coefficient de rafale de 5 produit par un vent moyen de $5 \mathrm{~m} / \mathrm{s}$ et une rafale de $25 \mathrm{~m} / \mathrm{s}$. Ensuite, les vents ont été classés en quatre catégories, en fonction de leur direction (Nord, Sud, Est, Ouest)

Enfin, les coefficients de rafale ont été calculés à la fois pour des données toutes les 15 minutes, et pour des données horaires, afin de pouvoir comparer nos résultats avec des travaux antérieurs [8].

Nous avons obtenu des coefficients de rafale dans un intervalle allant de 1,1 à 8,8 avec une moyenne de 1,8 . Ces valeurs sont parfois plus grandes que celles obtenues dans des expériences antérieures (valeurs inférieures à 4 pour Deaves, 1993). Cependant, nous avons trouvé assez peu de gros coefficients au cours de l'hiver, et en moyenne, le coefficient de rafale est de l'ordre de 1,8. Néanmoins, la plupart des calculs des coefficients de rafale effectués auparavant l'ont été pour des sites situés au niveau de la mer [8] et [9], alors que nous travaillons sur un site de haute montagne. Par ailleurs, nous avons constaté que la valeur du coefficient de rafale dépend fortement de la direction du vent. En effet pour les vents de Nord et de Sud, la probabilité P d'avoir un coefficient de rafale plus grand que 2 est de l'ordre de $10 \%$, alors que pour les vents d'Est ou d'Ouest, cette probabilité est supérieure à $40 \%$ (cf. figure 9). Cette forte différence est certainement à relier à la géométrie du Col du Lac Blanc, et à l'effet de canalisation Nord Sud des vents. Ce dernier résultat doit être analysé avec prudence, compte tenu de la faible quantité de vents d'Est par rapport aux vents de Nord, sur l'ensemble de l'hiver (53 direction Est pour le vent horaire, contre 1139 pour les vents de Nord).

La probabilité de dépassement, qui correspond à la probabilité d'avoir un coefficient de rafale supérieur à une valeur donnée G, est représentée sur la figure 9 .

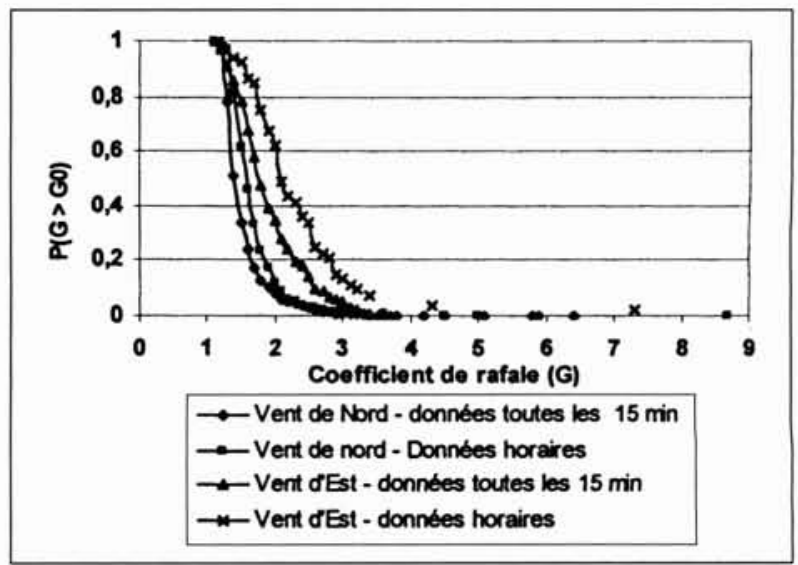

9. Probabilité de dépassement pour les vents de Nord et d'Est, pour le site de la Muzelle. 
Elle est généralement plus grande pour des données horaires que pour des données toutes les 15 minutes. Pour le vent, elle suit une loi de distribution de type Fisher-Tippet [8], qui peut s'écrire :

$$
P(\text { Rafale }>G)=1-\exp (-\exp (\mathrm{a}(\mathrm{G}-\mathrm{G} 0)))
$$

Où a est un coefficient de dispersion, et G0 est un coefficient de rafale de référence.

Nos résultats (cf. figure 10) mettent en évidence deux catégories de données, séparées par un nœud, pour un coefficient de rafale de 5,5 environ. Ceci est similaire aux résultats trouvés par Deaves [8], même si ses résultats correspondaient à des valeurs plus faibles du coefficient de rafale (nœud aux alentours de 2,0).

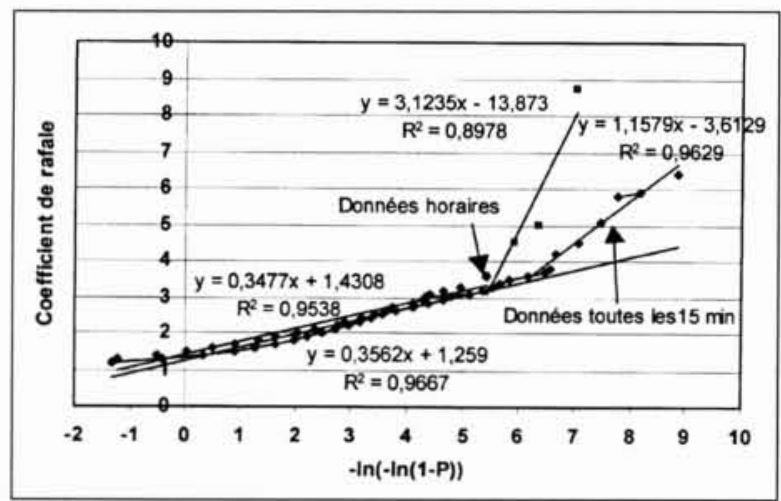

10. Loi de Fisher-Tippet pour les coefficients de rafale correspondant aux vents de Nord, pour le site de la Muzelle.

Par ailleurs, nous avons analysé chacune des périodes de vent qui générait de gros coefficients de rafale. Cela nous a permis de définir deux types de situations météorologiques. Le premier type correspond à des valeurs de l'ordre de 3 à 4 . Ils sont produits durant des événements sans variation de la direction du vent. Ils traduisent une diminution de la vitesse moyenne du vent, parallèlement à une augmentation ou à une faible diminution de la vitesse maximale. Le second type concerne des périodes où le vent change de direction. Ces événements génèrent les plus gros coefficients de rafale, de 5,5 à 8,8 . Les plus grands correspondent généralement à des changements de direction du vent du Sud vers le Nord.

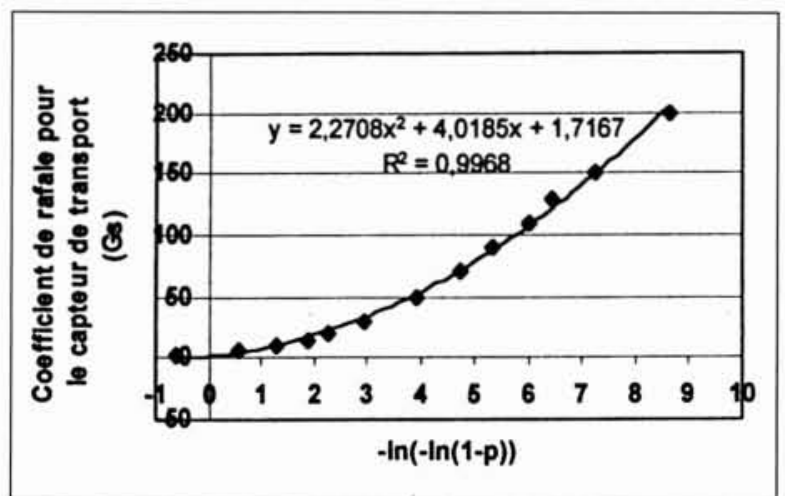

11. Test de la loi de type Fisher-Tippet pour le capteur acoustique de transport $n^{\circ} 5$ du site de La Muzelle. (P est la probabilité de dépassement).

\subsubsection{Coefficient de rafales du transport de neige par le vent}

Comme nous l'avons vu dans le paragraphe précédent, le coefficient de rafale du vent fournit de l'information sur l'aspect non stationnaire du vent. De la même manière, nous avons calculé le coefficient de rafale correspondant au signal enregistré sur le capteur acoustique de transport de la neige par le vent. II s'agit du rapport : Gs = signal maximal / signal moyen .

Nous avons effectué ce calcul à partir des données du capteur acoustique $n^{\circ} 5$ du site de La Muzelle, ce capteur étant situé dans une zone d'érosion (cf. figure 7) peu propice à l'enneigement. Nous avons tout d'abord filtré le signal moyen supérieur à $5 \mathrm{mV}$, après suppression de l'offset $(45 \mathrm{mV})$, car les valeurs faibles du signal ne correspondent pas à du transport, mais plutôt à du bruit parasite. Ensuite, le coefficient de rafale a été calculé toutes les 15 minutes sur l'ensemble des données de l'hiver.

Nos résultats montrent que la probabilité de dépassement de Gs ne suit pas une loi de type Fisher-Tippet, (cf. figure 11).

Nous avons mis en évidence deux types de phénomènes de transport.

Un premier type de transport est produit durant des rafales de vent ponctuelles. Il correspond à la zone 1 de la figure 12 et à la partie $\mathrm{A}$ de la figure 13. Ces épisodes génèrent de gros coefficients de rafale de vent, preuve de la présence de fortes rafales de vent. Par contre, ils ne produisent que très peu de signal moyen sur le capteur de transport, et des coefficients de rafale de transport également faibles. Par conséquent, ces épisodes ne produisent pas de transport de neige par le vent important, mais seulement des phénomènes sporadiques. Il en va de même pour la zone 3 de la figure 12, qui correspond à la zone $\mathrm{B}$ de la figure 13 : là encore le transport moyen est faible donc l'épisode de transport est peu important, même si ponctuellement, de gros coefficients de rafale de transport peuvent être générés.

Un deuxième type de transport beaucoup plus important est produit durant des épisodes de forte vitesse moyenne de vent. Ces périodes correspondent à la zone 2 de la figure 12 et à la partie $\mathrm{C}$ de la figure 13. Elles se produisent avec de faibles coefficients de rafale de transport. Cela s'explique par le fait que le capteur de transport ne peut générer des tensions supérieures à $5000 \mathrm{mV}$ (seuil de saturation du capteur). Par conséquent, si la tension moyenne est supérieure à $100 \mathrm{mV}$, ce qui est le cas durant un épisode de transport important, alors le coefficient de rafale de transport ne peut pas dépasser 50 .

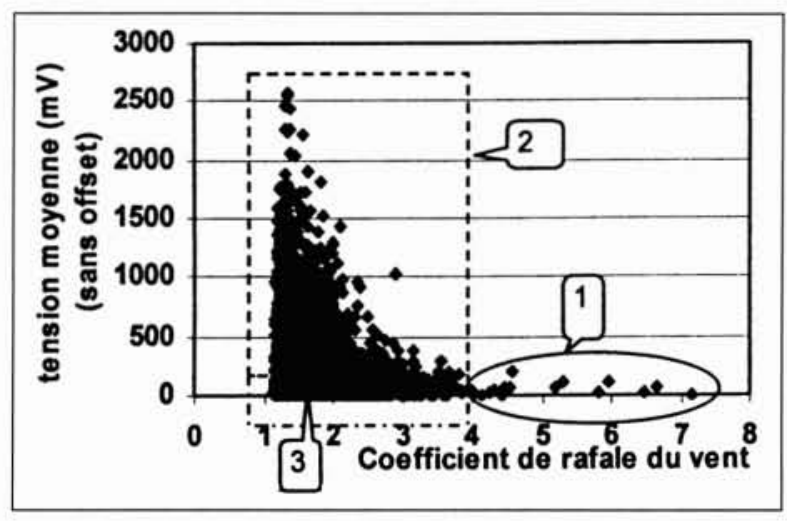

12. Signal moyen du capteur acoustique de transport $\mathbf{n}^{\circ} \mathbf{5}$, sur le site de La Muzelle. 


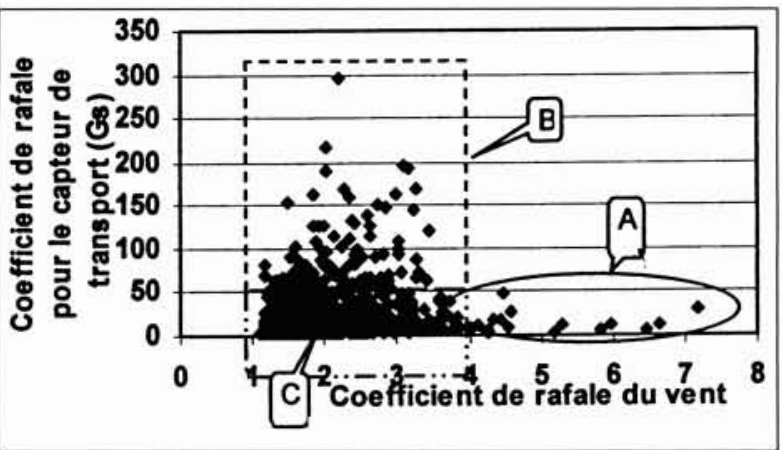

13. Coefficient de rafale de transport enregistré sur le capteur acoustique de transport $n^{\circ} 5$, sur le site de La Muzelle.

Par conséquent, l'étude du coefficient de rafale de transport montre que le transport est plus important quand il est généré par un vent régulier suffisamment fort que lorsqu'il est provoqué par des rafales de vent ponctuelles. Cependant, il faut utiliser ces résultats avec précaution. En effet, l'intensité du signal sur le capteur de transport ne dépend pas seulement du flux de particules frappant le tube, mais aussi de paramètres tels que le type de grain de neige et la hauteur de neige au sol (cf. paragraphe 3.2).

\section{IV — MODÈLE NUMÉRIQUE DE TRANS- PORT DE NEIGE PAR LE VENT}

Le modèle numérique utilisé a été décrit dans [1]. Ce modèle a été mis au point pour simuler le transport de la neige par le vent en représentant les deux couches de saltation et de diffusion turbulente. Il établit un bilan de masse et de quantité de mouvement du fluide et des particules dans chaque maille du domaine étudié en tenant compte du flux d'érosion et de dépôt et de l'interaction entre la phase fluide et la phase solide. Ce modèle a été testé pour l'étude de l'accumulation de particules solides autour d'une barrière en soufflerie. Dans ce cas, les paramètres d'entrée du modèle (vitesse de chute, vitesse de frottement, vitesse de frottement seuil) étaient connus et constants ; les résultats obtenus étaient plutôt satisfaisants. Le cas présent est cependant plus complexe. Comme mentionné précédemment, tous les paramètres d'entrée du modèle varient pendant l'épisode. Durant I'hiver 1998-1999, cinq périodes de transport ont été sélectionnées. La direction du vent est restée constante pour seulement deux d'entre elles, durant lesquelles on a observé des chutes de neige, ce qui complique la résolution du problème (la quantité de neige transportée ne dépend pas uniquement de la vitesse du vent (cf. figure 15)). Nous avons néanmoins essayé de reproduire l'évolution de la hauteur du manteau neigeux pour l'une de ces périodes, du 26 janvier au 1er février 2000. Durant cette période, la tempête a duré 6 jours avec 4 jours de chute de neige (cf. figure 14) (la hauteur totale de la chute de neige a été de 1,2 mètre). Dans l'état actuel de nos connaissances, nous ne pouvons utiliser les résultats du capteur acoustique de transport pour déterminer le débit de neige transportée. Nous n'utiliserons donc que les données de l'anémomètre et prendrons les formulations empiriques de Pomeroy [10] pour déterminer les quantités de neige transportées.

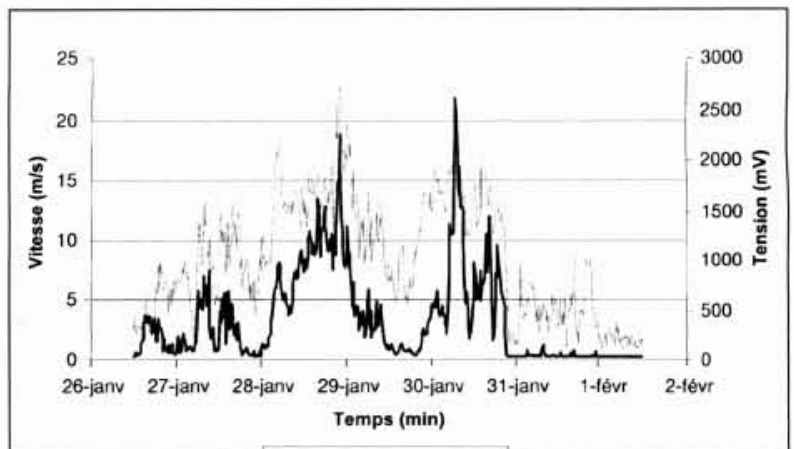

14. Tension moyenne sur $15 \mathrm{~min}$ (données issues du capteur acoustique $n^{\circ} 5$ ) et vitesse moyenne du vent sur $15 \mathrm{~min}$ du 26 janvier au ler février 1999.

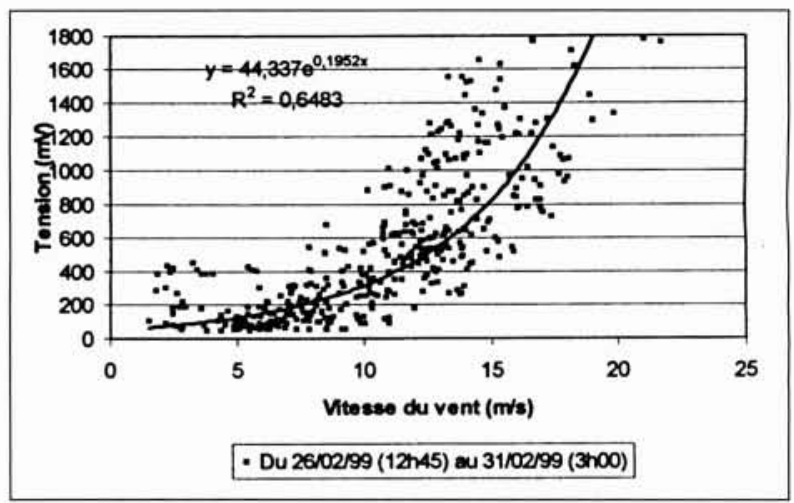

15. Tension moyenne sur $15 \mathrm{~min}$ (données issues du capteur acoustique $n^{\circ} 5$ ) en fonction du la vitesse moyenne du vent sur 15 min du 26 au 31 janvier 1999.

La simulation numérique sur cette longue période en utilisant le modèle couplant le vent et le transport de la neige par le vent n'est pas réalisable à partir de nos moyens de calcul. Nous nous sommes donc orientés vers la simulation de l'écoulement du vent utilisant la topographie et la vitesse turbulente à l'entrée du domaine $\left(\mathrm{u}_{*_{0}}\right)$. Nous avons ensuite déterminé la vitesse turbulente correspondante $\mathrm{u}_{*_{0}}(\mathrm{x})$ près de la surface du sol, dans tout le domaine. Puisque l'écoulement est proche de l'écoulement d'une couche limite turbulente avec une turbulence pleinement développée et comme l'évolution la vitesse moyenne mesurée durant la tempête à l'entrée du domaine changeait relativement lentement, nous avons supposé que lorsque la vitesse turbulente à l'entrée $\mathrm{u}_{{ } 0}$ changeait en $\mathrm{u}_{v}(\mathrm{t})$, la vitesse turbulente résultante dans l'ensemble du domaine $\mathrm{u}_{z}(\mathrm{t}, \mathrm{x})$ pouvait être déterminée par :

$$
\mathrm{u}_{*}(\mathrm{t}, \mathrm{x})=\mathrm{u}_{*_{0}}(\mathrm{x}) \cdot\left(\mathrm{u}_{* 0}(\mathrm{t}) / \mathrm{u}_{* 0}\right)
$$

En prenant la vitesse mesurée in situ à l'entrée du domaine comme condition à la limite amont, et en intégrant le flux moyen de chute de neige, nous avons appliqué le modèle d'érosion, de transport et de dépôt [1] à cet épisode.

Les résultats sont tracés sur la figure 16. Sur l'axe de gauche sont reportées les altitudes avant et après la tempête. Sur l'axe de droite sont reportées les accumulations obtenues par simulation et par mesures in situ. En comparant les résultats numériques et les mesures expérimentales, nous pouvons conclure que le modèle numérique reproduit assez bien la localisation du dépôt. Sur l'un des points, l'erreur reste tout de 
même relativement importante puisqu'elle avoisine les $50 \%$. La manière avec laquelle la vitesse a été déterminée sur l'ensemble du domaine peut expliquer cette erreur. Ce travail numérique va donc se poursuivre en utilisant le modèle total couplé pour obtenir de meilleurs résultats.

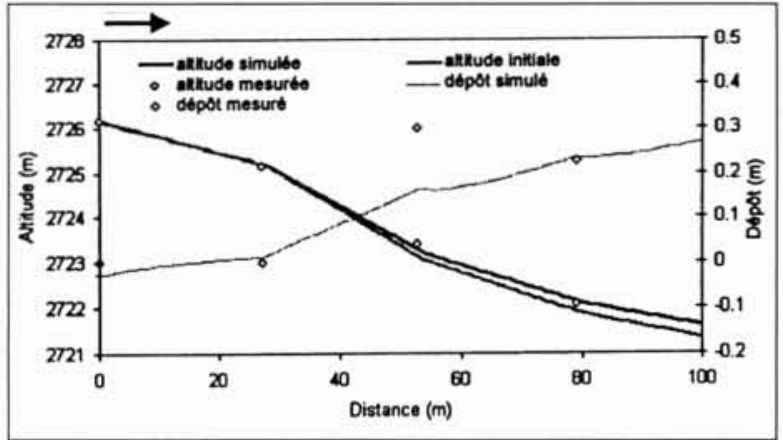

16. Modélisation numérique du transport de neige par le vent sur le site de La Muzelle du 26 janvier au 1er février 1999.

\section{$\mathrm{V} \square$ CONCLUSIONS}

Grâce à notre site expérimental de haute altitude du Col du Lac Blanc, nous avons pu créer une base de données bien documentée sur le vent, les épisodes de transport, et l'évolution des couches de neige du manteau. En particulier, nous avons trouvé des résultats intéressants concernant les coefficients de rafale, qui peuvent être plus grands pour le vent sur notre site expérimental que les valeurs de la bibliographie. De plus, grâce au capteur acoustique de transport de neige par le vent, nous avons mis en évidence le fait qu'un épisode de transport important se produit en général plutôt durant une période de vent fort et régulier que lors de fortes rafales de vent ponctuelles. Par ailleurs, la base de données que nous avons réalisée nous a permis de tester sur des données in situ le modèle numérique de transport de la neige par le vent développé au Cemagref. Pour améliorer encore ce modèle, il faudrait maintenant pouvoir introduire des mesures du flux de neige transportée comme paramètre d'entrée du modèle. Ce flux était en effet jusqu'à présent cal- culé au moyen d'une formule empirique utilisant la vitesse de vent mesurée ainsi que des vitesses seuils. Pour ce faire, nous devons calibrer le capteur acoustique, afin de pouvoir corréler le signal enregistré sur le capteur avec le débit de transport de la neige par le vent.

\section{Remerciements}

Cette étude n'aurait pas été possible sans le soutien, tant pour l'installation technique que pour la maintenance et la participation aux campagnes de mesures, de Frédéric Ousset (Cemagref), Philippe Pugliese (CEN) et Jean-Michel Panel (CEN).

\section{RÉFÉRENCES}

[1] NaAim M.. F. NaAim-Bouvet and H. Martinez. 1998. Numerical simulation of drifting snow : erosion and deposition models. Ann. Glaciol. 26. 191-196.

[2] MelLor M. and G. Fel..ers. 1986. Concentration and flux of windblown snow. Special Report 86-11. CRREL.

[3] Nanim-Bouvet F., M. Naaim and H. Martinez. 1996. Profils de concentration de la neige soufflée : théorie, résolution et validation expérimentale in situ. La Houille Blanche, 5, 53-57.

[4] Durand, Y.E. Brun, L. Merindol. G. Guyomarc'h and E. Martin. 1993. A meteorological estimation of the relevant parameters for snow models. Ann. Glaciol., 18, 65-71.

[5] Brun E., P. David, M. SUdUL and G. Brunot. 1992. A numerical mode to simulate snow-cover stratigraphy for operational avalanche forecasting. J. Glaciol., 38(128), 13-22.

[6] Dyunin A. K.. Kotlyakov V. M.. 1980. Redistribution of snow in the mountains under the effect of heavy snow-storms. Cold region Science and Technology, 3, 287-294.

[7] GuYomarc'H G. and L. Merindol. 1998. Validation of an application for forecasting blowing snow. Ann. Glaciol.., 26, 138-143.

[8] DeAves D. M.1993. Analysis of gust factors for use in assessing wind hazard. Journal of wind Engineering and Industrial Aerodynamics, 45 , $175-188$

19] Béranger, M. and G. Pages. 1958. Contribution à l'étude des rafales. Monographies de la Météorologie Nationale, $n^{\circ} 10$

[10] Pomeroy, J.W, and D.M. Gray, 1990. Saltation of Snow. Water Resor. Res. 26(7), 1583-1594 